\title{
Cooperation in wild Barbary macaques: Factors affecting free partner
}

choice

Sandra Molesti ${ }^{\mathrm{a}, 1}$ and Bonaventura Majolo ${ }^{\mathrm{a}}$

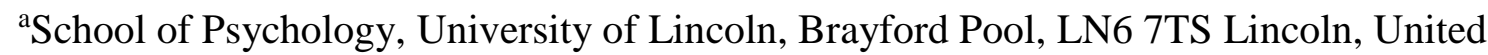

Kingdom

Journal Animal Cognition:

Molesti, S. \& Majolo, B. 2016. Cooperation in wild Barbary macaques: factors affecting free partner choice. Animal Cognition, 19, 133-146.

${ }^{1}$ To whom correspondence should be addressed: Sandra Molesti

Phone number: (0033)782368438

E-mail: sandra.molesti@outlook.com 


\section{Abstract}

A key aspect of cooperation is partner choice: choosing the best available partner improves the chances of a successful cooperative interaction and decreases the likelihood of being exploited. However, in studies on cooperation subjects are rarely allowed to freely choose their partners. Group-living animals live in a complex social environment where they can choose among several social partners differing in, for example, sex, age, temperament, or dominance status. Our study investigated whether wild Barbary macaques succeed to cooperate using an experimental apparatus, and whether individual and social factors affect their choice of partners and the degree of cooperation. We used the string pulling task that requires two monkeys to manipulate simultaneously a rope in order to receive a food reward. The monkeys were free to interact with the apparatus or not and to choose their partner. The results showed that Barbary macaques are able to pair up with a partner to cooperate using the apparatus. High level of tolerance between monkeys was necessary for the initiation of successful cooperation, while strong social bond positively affected the maintenance of cooperative interactions. Dominance status, sex, age and temperament of the subjects also affected their choice and performance. These factors thus need to be taken into account in cooperative experiment on animals. Tolerance between social partners is likely to be a prerequisite for the evolution of cooperation.

Keywords: cooperation, tolerance, macaque, partner choice, social bond, cognition 


\section{INTRODUCTION}

Cooperation is generally defined as two or more individuals acting together to achieve a common goal (Boesch and Boesch 1989). In particular, mutual cooperation (also called mutualism; de Waal 2000) defines cooperative interactions that yield benefits simultaneously to the participants involved. Cooperation may involve different levels of cognitive abilities, ranging from simple synchronous actions to a division of labour among participants (Boesch and Boesch 1989). Although simultaneity is an essential proximate mechanism of mutual cooperation, comprehension of the partner's role would allow individuals to actively coordinate their actions with those of their partner, facilitating joint action (Melis and Semmann 2010).

The performance of animals in solving a cooperative task has been investigated using a classic cooperative experiment (Crawford 1937) where two animals have to simultaneously pull two ropes to bring within arm's reach a box containing food. Different versions of this experiment have demonstrated success in simultaneous pulling in a variety of primate species (e.g. Mendres and de Waal 2000; Chalmeau et al. 1997; Cronin et al. 2005; Hirata and Fuwa 2007; Hare et al. 2007), but also, more recently, in non-primate species such as rooks (Corvus frugilegus; Seed et al. 2008; Scheid and Noë 2010), African grey parrots (Psittacus erithacus; Péron et al. 2011), hyenas (Crocuta crocuta; Drea and Carter 2009), and Asian elephants (Elephas maximus; Plotnik et al. 2011). Furthermore, in experiments where the arrival of the partner at the pulling apparatus was artificially delayed, some species (e.g. chimpanzees and elephants; Melis et al. 2006a; Plotnik et al. 2011) were able to wait the arrival of their partner before pulling, which suggests that the animals understood the need of their partner to solve the task. Conversely, rooks and parrots were unable to inhibit their pulling before the arrival of their partner (Seed et al. 2008; Péron et al. 2011). The string pulling tasks have several advantages in terms of ecological validity and research questions that can be addressed. Pulling tasks are similar to natural resource acquisitions in the natural habitat of non-human primates 
and other taxa (e.g. pulling branches toward self to reach fruits and leaves or pulling to remove bark from a tree). These tasks also have the advantage of both visual and kinesthetic feedback to animals in the course of the simultaneous action, which may facilitate the understanding of the cause and effect relationship of the mechanism by the subjects (Mendress and de Waal 2000; de Waal and Suchak 2010).

Partner choice is key to initiate and maintain cooperative behaviours (Noë 2006): choosing the best available partner (e.g. in term of reliability or ability to perform a task) improves the chances of successful cooperation and decreases the likelihood of being exploited (Noë 2001; 2006). However, studies where the subjects were allowed to freely choose their partners to solve the cooperative task are rare. For example, chimpanzees were able to recruit a cooperative partner (by unlocking a door) only when solving the problem required cooperation, and they recruited the most efficient partner (chosen between two available individuals) based on their experience with each of them on a previous day (Melis et al. 2006a). In a recent study, captive chimpanzees spontaneously initiated and maintained a high level of success in solving cooperative tasks when given the choice of freely choosing their partners (Suchak et al. 2014). Group-living animals live in complex social environments where they can choose among several potential social partners differing in, for example, sex, age, temperament, dominance status, or strength of social bonding. The capacity to make effective choices among potential social partners (i.e. reducing the risk of exploitation and increasing the chance of cooperation) is thus an important social skill.

Tolerance between partners is expected to increases the chances of successful cooperation (e.g. Melis et al. 2006b; Hare et al. 2007). According to this hypothesis, more tolerant species would perform better in cooperative tasks than less tolerant species (e.g. Petit et al. 1992; Hare et al. 2007). For example, because of their high social tolerance, Tonkean macaques (Macaca tonkeana; Petit et al, 1992) were more likely to simultaneously push heavy 
stones to retrieve food compared to less tolerant primate species (Fady, 1972; Burton, 1977; Petit et al, 1992). Similarly, higher level of tolerance while co-feeding allowed bonobos to outperform chimpanzees on a cooperative task where the food reward was highly monopolisable (Hare et al, 2007). Tolerance is also expected to explain differences in cooperation across dyads, especially when animals are tested in tasks requiring joint effort. In chimpanzees, dyads that were more willing to share food outside the cooperative task, were better able to simultaneously pull two ropes to get food (Melis et al. 2006b). The performance of two rooks in solving a cooperative task was higher when within-dyad tolerance was higher (Seed et al. 2008). Moreover, there is a growing body of evidence showing that the strength of social bond between two animals positively affects cooperative behaviours, as strong social bonds would reduce the uncertainty about the partner response (Noë 2001; Silk 2003; Schino and Aureli 2009; Cronin 2012).

Factors such as the age, sex, dominance status and temperament of the subjects can affect their choice of partner and their performance in solving cooperative tasks. Individuals may be less willing to cooperate with higher ranking partners, which often monopolise experimental apparatuses that dispense food (Chalmeau and Gallo 1993; de Waal and Davis 2003; Drea and Carter 2009; Suchak et al. 2014). The temperament of the subjects may also affect cooperation (Bergmüller et al. 2010; McNamara and Leimar 2010). In rooks, bolder birds were more willing to approach the experimental apparatus than shier birds, and subjects performed better in a cooperative task when paired with a bolder than shier partner (Scheid and Noë 2010). Furthermore, capuchins (Mendres and de Waal 2000) and cotton-top tamarins (Hauser et al. 2003) performed better in a cooperative task with same-sex than different-sex partners (but see Pansini 2011). Finally, whilst the age of the subjects did not affect their cooperative performance in chimpanzees (Melis et al. 2006b) and wild vervet monkeys (Pansini 2011), the effect of age class (i.e. adult or juvenile) on partner choice for cooperation was rarely 
tested. Therefore, each of these factors may affect the performance and choice of partners in cooperative task, and their relative role in affecting cooperation need to be investigated.

The aim of our study was to investigate the factors influencing mutual cooperation in a partially provisioned group of wild Barbary macaques (Macaca sylvanus). We presented the monkeys with a cooperative apparatus; the animals could choose to interact or not with it and they had the opportunity to freely choose their cooperative partners among their group mates (i.e. partners were not artificially paired up). To solve the task, two monkeys had to manipulate simultaneously the two ends of the same rope of the apparatus in order to bring within arm's reach a tray containing a food reward for each partner (Hirata and Fuwa 2007). The Barbary macaque is a relatively tolerant species (Thierry et al. 2004), yet also sharing some social features with more despotic macaque species such as low frequency of counter-aggression (Balasubramaniam et al. 2012) and a steep dominance hierarchy (Kaburu et al. 2012). Barbary macaques form coalitions, reciprocate grooming or inter-change it for other benefits, show food tolerance, and affiliate often with other group members of different age and sex (Widdig et al. 2000; Berghänel et al. 2011; Carne et al. 2011). Since these factors are expected to favour the occurrence of cooperation (or to represent forms of cooperation, as in the case of coalition formation), this species is of particular interest to experimentally assess cooperative behaviours. We first investigated whether wild Barbary macaques were able to cooperate using the apparatus or not, as this species has never been tested in cooperative experiments. Second, we aimed to assess which individual (i.e. sex, age class and temperament) and social factors (i.e. dominance status and the dyadic degree of tolerance and strength of social bonds) affected partner choice for the initiation of cooperation (i.e. with which partner individuals cooperated or not) and for the maintenance of cooperation (i.e. with which partner individuals cooperated the most). To our knowledge, this is the first study to use a string pulling cooperative apparatus to test cooperation in a wild macaque species. 


\section{METHODS}

\section{Study subjects}

This study was conducted on a partially provisioned group of wild Barbary macaques living in a deciduous cedar and oak forest of the Ifrane National Park, between 1600 and $1860 \mathrm{~m}$ a.s.l, in the Middle Atlas Mountains of Morocco. At the start of the data collection, the group was composed of 21 adults (including sub-adults, i.e. from 4-5 years old; 11 males and 10 females), 15 juveniles (between 1 and 3-4 years old; 7 males and 8 females) and several infants. Three juveniles died during the study period and were consequently not included in the analyses. The study group was chosen because it was partially provisioned by tourists and local people (Maréchal et al. 2011). The animals were often near a road cutting through their home range and could be approached very closely by tourists who fed the monkeys with a variety of food, such as fruits, bread and peanuts. Such level of provisioning allowed us to run experiments involving food with the monkeys without affecting their usual diet and behaviour. Permission to conduct this study was granted by the Haut Commissariat aux Eaux et Forêts et à la Lutte Contre la Désertification of Morocco and the Ethics Committee of the University of Lincoln, U.K.

\section{Data collection}

We collected data from November 2010 to January 2012. During the course of the study period the monkeys were free to approach and interact with the experimental apparatus. 


\section{Materials}

We adapted the Crawford's string pulling paradigm (1937) to use it with wild macaques in their natural environment. We used two identical boxes of $85 \times 35 \times 35 \mathrm{~cm}$ each. Each box contained a tray which could slide along the length of the box through a rail. A jar lid was fixed in the middle of each tray and used to contain food rewards. The tray could be brought within arm's reach, from the back to the front of the box by pulling a rope from the front of the box. A total length of $75 \mathrm{~cm}$ had to be pulled to bring the tray within arm's reach. The sides and roof of the boxes were made of clear Perspex thus the monkeys could easily monitor the effect of their behaviours on the movements of the tray, rope and food rewards. There was a hole at the front of each box to allow the monkeys to introduce one hand and grab the food reward when the tray was within arm's reach. We used slices of fruits, vegetables, or peanuts to bait the apparatus. The data collection was divided in two distinct time periods (see below). We first trained the study animals to use one box alone; when training was successfully completed we joined the two boxes together and used this experimental apparatus to investigate cooperation in Barbary macaques using a series of test and control sessions.

\section{Training}

We trained the monkeys between November 2010 and March 2011. During the training, only one box was used and one rope was directly attached to the tray. Through the shaping technique, the monkeys learned to use one box to get food, that is, to pull the rope to bring the tray within arm's reach and get the food reward placed on it. During the first phase of training, the tray was baited and placed within arm's reach at the front of the box. The monkeys had to introduce one hand into the front hole to reach the reward. This allowed the wild monkeys to become habituated to the box and associate it with food. During the second phase, the tray was baited but placed out of reach at the back of the box. The monkeys had to pull the rope to bring 
the tray within arm's reach and grasp the reward. A subject was considered trained when it performed the full successful sequence for a minimum of 15 times consecutively. Twenty one monkeys (7 males and 14 females, 14 adults including sub-adults and 7 juveniles) met this criterion during the training and were thus considered to be trained. It took between 1 and 29 sessions, from their first presentation of the apparatus to the first success, to the monkeys to learn to pull the rope in order to get the food (mean number of sessions per monkey $\pm \mathrm{SE}=9.19$ \pm 1.64). Five additional monkeys ( 3 males and 2 females, all juveniles) learned to use the apparatus (i.e. the two boxes joined together) during the testing period without being trained by the experimenter. Contrary to chimpanzees (Chalmeau and Gallo 1993), in our study higherranking males were not interested in the boxes and did not monopolise them during training or testing.

\section{Testing}

We conducted a series of test and control sessions from March 2011 to January 2012. The presentation of the cooperative and control tests were alternated during the study period. Individuals were free to interact with the apparatus and to choose their partner. For the test sessions, the two boxes were joined together at $215 \mathrm{~cm}$ apart (Fig. 1). Moreover, the two trays (i.e. one for each box) were attached together with a blue plank to form a longer tray across the boxes (Fig. 1). We used the 'loose string paradigm' of Hirata and Fuwa (2007). For this, a long rope went freely along the two boxes and tray, as well as through two pulleys placed on both extremities of the tray. The rope was $10 \mathrm{~m}$ long and $3 \mathrm{~m}$ of the rope extended in front of each box. Consequently, to solve the task (i.e. bring the tray within arm's reach) and both get a reward, two monkeys had to coordinate their movements and manipulate simultaneously both ends of the rope, either by pulling both ends, or by pulling one end and holding the other one. Following the 'loose string paradigm' of Hirata and Fuwa (2007), if only one monkey pulled 
one end of the rope, the rope moved freely through the pulleys and the tray did not move. However, to prevent the monkeys to run away with the rope after pulling, the experimenter blocked the rope before it came out of the box. Following this, the experimenter waited for the monkey to leave the apparatus and correctly repositioned the rope. Because of the distance between the two boxes it was not possible for one monkey alone to simultaneously manipulate the two ends of the rope. We placed food rewards on the lid inside each box: the food was always of the same kind and quantity across the two boxes. We baited the trays each time two monkeys succeeded to cooperate and finished consuming their reward. Although it was theoretically possible to bring the tray within arm's reach by pulling with both hands the middle of the blue plank (see Fig. 1), we never observed the monkeys pulling the plank.

During the control sessions, the two boxes were also joined together, but not the trays, and a rope was attached to each single brown tray (Fig. 1). Consequently, during control sessions the two trays worked independently and the monkeys did not need to act simultaneously to obtain food. We baited each tray anew when a monkey got the reward and finished consuming it. The apparatus was visually distinguishable between the test and control sessions: for the test sessions the apparatus contained a long blue tray across the two boxes, whereas for the control sessions the apparatus contained two single brown trays inside each box.

We made available to the monkeys the apparatus for test or control sessions for 40 minutes for each session, several times per day. Sessions were stopped when no monkey approached the apparatus for 10 minutes. A total of 45 control and 165 test sessions were conducted. Series of test and control sessions were alternated across days. However, we ran a larger number of test than control sessions because the monkeys needed more exposure to the test apparatus in order to learn to solve the task. The number of sessions per series varied depending on the motivation of the monkeys to manipulate the current apparatus (mean number 
of sessions per series $\pm \mathrm{SE}=13.1 \pm 3.7$ ). Control sessions also allowed to keep motivated the monkeys who did not succeed to cooperate during their initial interactions with the test apparatus. Because the apparatus was made freely available to the monkeys, the number of trials (i.e. when the apparatus was baited) per session differed greatly depending on the current activity of the monkeys with the apparatus during the session (i.e. the more the monkeys were successful, the greater the number of trials). Such flexibility, in the time and opportunities that we gave the study animals to interact with the apparatus and cooperate, was necessary due to the opportunistic nature of our data collection. In other words, since the monkeys were free to approach the apparatus, or not interact with it, when it was available, we could not control for the presence or absence of the monkeys near the apparatus, or for the time that each animal spent (or not) attempting to cooperate with other individuals.

During each session, we used focal sampling focusing on the apparatus to continuously record the ID and behaviours of each monkey interacting with the apparatus. For each monkey, we recorded all the occurrences of pulling or holding the rope in relation to the presence or absence of a partner. We defined 'holding the rope' as when an individual held the rope with one or both hand(s) for at least $5 \mathrm{sec}$ and without pulling it. We defined 'pulling the rope' as when an individual held the rope with one or both hand and made a pulling movement, that is, an exertion of force on the rope toward themselves. Two occurrences of behaviour had to be separated by at least $5 \mathrm{sec}$ to be considered as two separate events. We classified the pulling or holding behaviour of a monkey into categories in relation to the absence or presence of partner. 'Partner absent' was defined as a monkey pulling or holding the rope while no partner was present in front of the other rope (i.e. within $50 \mathrm{~cm}$ ). 'Partner present' was defined as a monkey pulling or holding the rope while a partner was within arm's reach of the other rope (the partner could be manipulating the rope or not). We never observed more than one partner present in front of the other rope. Additionally, we recorded the ID of the monkeys within $1.5 \mathrm{~m}$ all around 
the apparatus when a monkey was manipulating (i.e. pulling or holding) a rope and no partner was present in front of the other rope. When a monkey was manipulating a rope in the presence of more than one partner within $1.5 \mathrm{~m}$ all around the apparatus, we still considered this manipulation as only one event. For example, if a subject pulled a rope in the presence of two partners within $1.5 \mathrm{~m}$ of the apparatus, we considered this as one pulling event for that subject. Finally, we also recorded whether the tray was successfully brought within arm's reach or not. 'Cooperation' was defined as two monkeys manipulating simultaneously the two ends of the rope during the cooperative test and succeeding to bring the tray within arm's reach. Cooperation could be achieved by either two monkeys pulling simultaneously both ends of the rope, or by one monkey pulling one end while the partner firmly held the other end without pulling it. For each monkey, their first cooperation corresponded to the first time they solved the cooperative task.

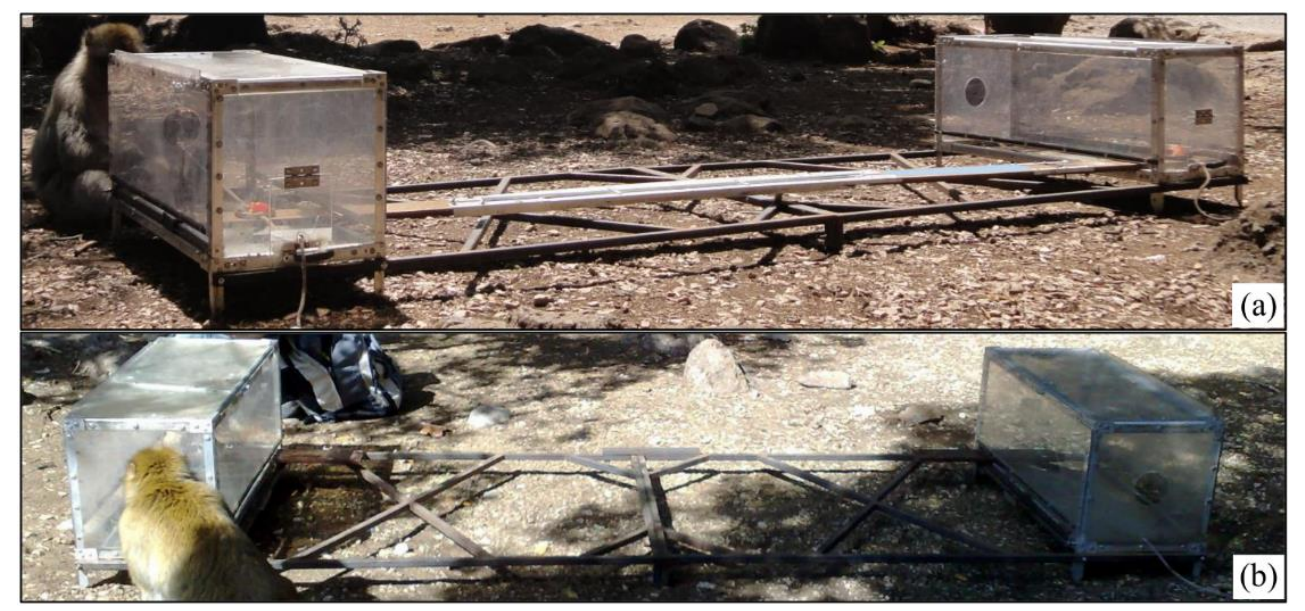

Fig. 1 Pictures from (a) the back of the cooperative test (with one long tray across the two boxes) and (b) the front of the control (with one independent tray inside each of the two boxes) (pictures by S. Molesti) 


\section{Social and individual factors}

We collected scan samples (Altmann 1974) every hour on the activity of all visible adult monkeys to assess the strength of social bond and tolerance level between the study animals. The activity of each study animal was not sampled more than once in a single scan. For each visible monkey and within 10 minutes of the beginning of the scan, we first recorded their proximity (i.e. $\leq 1.5 \mathrm{~m}$ but not grooming), or grooming exchanged, between the study animals or with other group members. We used these data to assess the strength of social bonds between the animals. Second, we also recorded the occurrence of co-feeding, defined as a monkey feeding within $2 \mathrm{~m}$ of another monkey, to assess the dyadic tolerance level between the animals. Finally we collected ad libitum (Altmann 1974) data opportunistically on any observed dyadic conflicts (i.e. one opponent displaying aggressive behaviour and the other opponent submission), in order to determine the dominance hierarchy of the monkeys. Our study was not initially planned to be conducted on juveniles as social relationships between juveniles may be difficult to define (Pereira and Fairbanks 2002), thus social bonding, tolerance, and dominance status between juveniles were not assessed. We added data on juveniles during the data analysis stage since juveniles often interacted with the apparatus and adults also cooperated with juvenile partners. Therefore, we integrated data on juveniles in the analyses in order to give a more comprehensive picture of how Barbary macaques cooperated using the experimental apparatus. Because the scan samples were collected on the activity of all visible adults in relation to all group members, this procedure allowed us to also gain data on the social interactions between adults and juveniles (i.e. dyadic degree of tolerance and strength of social bonds between adults and juveniles), but not between two juveniles.

Since the monkeys were used to human presence (Maréchal et al. 2011) and the experimenters were always present near the apparatus to place food reward and check its proper functioning, we choose to conduct 'flight distance test' (e.g. Stankowich and Coss 2006; Møller 
et al. 2008; Carrete and Tella 2010; Carter et al. 2010) to assess difference of temperament (i.e. shyness toward humans) among the study subjects. This test assessed at which distance a monkey would flee an approaching human. A temperament test started when a monkey was seating without any other monkey within $1.5 \mathrm{~m}$ around, and one experimenter was standing at $4 \mathrm{~m}$ in front of the monkey. The experimenter first coughed to have the monkey focused on their presence, and then walked straight toward the animal at stable pace, with no obstacles between the monkey and the experimenter. The test stopped when the subject moved away from the experimenter or if the monkey did not move, when the experimenter arrived at $40 \mathrm{~cm}$ in front of the monkey. We recorded the distance at which the subject started to move away from the experimenter. If the subject did not move at the end of the test, the distance of $40 \mathrm{~cm}$ was attributed to that monkey. The shorter the distance was, the bolder the monkey was considered to be. We conducted three temperament trials for each monkey.

\section{Data analysis}

\section{Social and individual factors}

We calculated a composite sociality index (hereafter CSI) to measure the strength of the social bond between two individuals, based on the data collected during 929 hourly scans. For each dyad, we calculated their CSI based on the formula (Silk et al. 2006):

$$
\frac{\sum_{\mathrm{i}=1}^{2} \frac{\mathrm{x}_{\mathrm{i}}}{\mathrm{m}_{\mathrm{i}}}}{2}
$$

$\mathrm{x}_{\mathrm{i}}=$ dyad's value for each of the two behavioural measures (i.e. the proportion of hourly scans in which two monkeys of a dyad were grooming, or in proximity, divided by the total number of scans in which the general activity of the two animals was recorded).

$\mathrm{m}_{\mathrm{i}}=$ group's mean value for the proportion of hourly scans spent grooming, or in proximity, by the group. 
A high CSI indicates a high quality relationship between the two monkeys of the dyad. The CSI values ranged from 0 to 16.1 (mean CSI value $\pm \mathrm{SE}=1 \pm 0.07$ ). Dyads with a CSI value above the group's mean were considered having a strong social bond, whereas dyads with a CSI value under the group's mean were considered having a weak social bond. We calculated co-feeding frequency as the proportion of hourly scans in which two monkeys were within $2 \mathrm{~m}$ and at least one monkey was feeding, divided by the total number of scans in which the general activity of the two monkeys was recorded. Co-feeding frequencies ranged from 0 to 0.13 (mean co-feeding frequency $\pm \mathrm{SE}=0.01 \pm 0.001)$. Dyads with a co-feeding frequency above the group's mean were considered highly tolerant, whereas dyads with a co-feeding frequency under the group's mean were considered having a low tolerance level.

Based on the dyadic conflicts collected ad libitum, we constructed a winner-loser sociometric dominance matrix. We used Matman 1.1 (Noldus Information Technology 2003; de Vries et al. 1993) to assign an ordinal dominance rank to each adult. Since their rank position within the group changes frequently until they reach adulthood, all juveniles were assigned the same lowest rank of the group, and juvenile-juvenile data were not considered in the analyses on rank distance. The absolute value of the rank difference between two monkeys was calculated for each dyad, except for dyads of two juveniles. The rank distances ranged from 1 to 21 (mean rank distance $\pm \mathrm{SE}=9.33 \pm 0.27$ ). Dyads with a rank distance under the group's rank distance mean were considered as 'close-rank' partners whereas dyads with a rank distance above the group's rank difference mean were considered as 'distant-rank' partners.

Based on the temperament tests, we calculated the mean distance at which the experimenter could approach a monkey before it fled for each monkey across the three tests. The distances ranged from 40 to $216.7 \mathrm{~cm}$ (mean distance $\pm \mathrm{SE}=87.8 \pm 8.2$ ). Individuals with a distance under the group's mean distance were considered as bold whereas individuals with distance above the group's mean distance were considered as shy. Additionally, we calculated 
the absolute value of the difference of distances between two monkeys for each dyad of the group. Smaller difference of distance indicated that the two monkeys were of more similar temperament.

Although the measures of CSI and tolerance were positively correlated (Partial Kendall rowwise matrix correlations: $\tau_{r w}=0.38, p=0.0001, N=462$ ) we kept both measures as two different factors in the analyses to provide a more comprehensive description of partner choice. Indeed, while the CSI index indicates the general strength of the social bond between two monkeys, including the time spend together and grooming, tolerance focuses more precisely on acceptance between two monkeys when feeding. Therefore these two factors may affect differently cooperative behaviours involving food.

\section{Experiments}

\section{Performance}

We recorded a total of 135 hours of focal sampling during the testing period. We never observed more than one monkey manipulating each rope, so we never observed cooperation between more than two individuals at a time.

We ran a first set of analyses to assess whether the monkeys learned that they needed to cooperate with a partner to obtain food in the test session but not in the control. For each individual, we calculated the percentage of, respectively, pulling or holding the rope, occurring in the presence of a partner versus when a partner was absent. First, the percentage of manipulations (i.e. holding or pulling) in the presence of a partner for the test session was compared before and after the monkeys first solved the cooperative test. If the monkeys learned that a partner was needed to solve the cooperative test, they would manipulate the rope next to a partner more often after than before their first cooperation. Then, the percentage of manipulations in the presence of a partner after the monkeys first cooperated was compared 
between the test and control sessions. If the monkeys differentiated between the mechanism of the test session, which required cooperation with a partner, and the control session, which did not, they would manipulate the rope in the presence of a partner more often in the test than in the control, after their first successful cooperation in the test. Finally, for the test sessions and after the monkeys' first cooperation, the percentage of manipulations in the presence of a partner was compared with the percentage of manipulations in the absence of a partner. After the monkeys learned that a partner was needed to solve the cooperative test, they should refrain from manipulating the rope when no partner was directly available in the test session. We used Wilcoxon signed rank tests for paired samples and two-tailed exact p-values to compare individual percentages.

We ran a series of analyses to investigate whether the performance of the monkeys increased over time during the test, and whether they remained stable during the control. We analysed the rate of success of the individuals across the sessions during the test and the control sessions. First, for each session and for each subject, we calculated the rate of success by dividing the number of successes (i.e. the events of cooperation for the test and events of pulling alone the tray within arm's reach for the control) by the total number of manipulations. For each session, we calculated the mean of individual success rate. We ran two Spearman correlations between the rate of success and the session number; one for the test and one for the control.

Factors affecting the initiation of cooperation

We investigated the initiation of cooperative interactions by analysing the tendency of monkeys to cooperate at least once with particular partners but not with others. For this, we tested the factors affecting the probability of a dyad to cooperate at least once or not among all of the monkeys trained using a generalised linear mixed model (GLMM) with a logistic distribution. Each potential dyad of trained monkeys was treated as a single data point in the 
model. Note that dyads of two juveniles were not considered in this analysis as data on social bonds, tolerance and dominance were not available among juveniles. The risk of sample inflation was controlled for by adding to the model the ID of the two monkeys as crossed random factors (Pinheiro and Bates, 2000). The binary dependent variable was whether the dyads cooperated at least once during the test sessions or not. Since events of cooperation were collected opportunistically (i.e. each monkey might have not been available during every session), we could not use the number of cooperation performed in this analysis. The test variables were the CSI value, co-feeding frequency, difference of rank status within the dyad, difference of temperament within the dyad, sex (i.e. same-sex or different-sex), and age class (i.e. same-age class or different-age class) of the dyads.

Factors affecting the maintenance of cooperation

We investigated the maintenance of cooperative interactions by analysing which social and individual factors affected the level of cooperation in the test sessions. For this, we examined whether, among all of the cooperation performed by each animal, monkeys cooperated more often with particular partners than with others. Among all of the events of cooperation performed by each individual, we calculated the percentage of cooperation performed in a series of three pairwise comparisons, namely, percentage of cooperation with partners with whom monkeys had a strong versus weak social bond, with a high versus low level of tolerance, and with close-rank versus distant-rank partners. Cooperation between juveniles was not considered for these analyses, so three juveniles were excluded as they only cooperated with juveniles. Moreover, we also ran six pairwise comparisons to test whether the sex, age class, or temperament of the individuals affected the level of cooperation observed. We used Wilcoxon signed rank test for paired sample and two-tailed exact p-values to compare the percentage of cooperation. 
We investigated whether the social and individuals factors affected the pulling behaviour of the monkeys during the control. To this end, we analysed the successful pulling behaviour during the control (i.e. one monkey pulled the rope, making the tray within arm reach) according to the presence or absence of another individual in front of the other box (the two boxes and trays being independent). First, we analysed whether the monkeys pulled more when another monkey was present in front of the other box or when no other monkey was present. We never observed more than one monkey present in front of the other box. For each individual and among all the events of successful pulling, we calculated the percentage of pulling performed when another individual was present in front of the other box versus when no other monkey was present. Second, during the control and when the monkeys successfully pulled in the presence of another monkey in front of the other box, we looked at whether they pulled more in the presence of a particular type of partner than another, following the same series of pairwise comparisons undertaken for the test (i.e. the effect of social bonding, tolerance, rank, age class, sex, and temperament). We used Wilcoxon signed rank tests for paired samples and two-tailed exact p-values to compare the percentages.

For each box plot presented below, the bottom and top sides of the boxes represent respectively the first and third quartiles of the data, the band inside the boxes represents the median, and the bottom and top ends of the whiskers represent respectively the minimum and maximum scores of the data set.

\section{RESULTS}

Among all the manipulations of the rope recorded, pulling the rope and holding the rope represented respectively $98.1 \%$ and $1.9 \%$ of the events during the test, and $99.8 \%$ and $0.2 \%$ of the events during the control. Among the 26 monkeys trained, 84.6\% (10 males and 12 females, 
10 adults and 12 juveniles) succeeded at least once to cooperate, that is, to pair up with a partner to bring the tray and the rewards within arm's reach (Table 1). In total, cooperation was performed by 93 pairs of monkeys (among these 93 pairs, cooperation was performed $32.3 \%$ by juvenile-juvenile pairs, $51.6 \%$ by juvenile-adult pairs and $16.1 \%$ by adult-adult pairs), out of 325 potential pairs (i.e. $28.6 \%$ of the potential pairs). The number of cooperation ranged from 1 to 391 cooperation per monkey (mean $\pm \mathrm{SE}=94.82 \pm 20.22$ ), with a total of 800 cooperation recorded. The number of cooperative partners per monkey ranges from 1 to 15 partners (mean $\pm \mathrm{SE}=8.5 \pm 1.01$ ). The cooperation was performed by pulling simultaneously both ends of the rope at $95.4 \%$, while $4.6 \%$ were performed by one monkey pulling one end of the rope while its partner firmly held the other end of the rope. No subject cooperated the first time they were presented with the cooperative test. The dominant individuals who were not interested in the apparatus always kept a distance of more than 5 metres from the apparatus and were never observed eating the reward of monkeys who succeeded to manipulate the apparatus. Among the individuals trained, we observed a monkey eating the reward of their partner in only $1.25 \%$ of the cooperative interactions. 
Table 1 Characteristics (age, sex and dominance status within the group) of the individuals who succeeded to cooperate, as well as their percentage of cooperation performed with partners with strong social bond versus partners with weak social bond

\begin{tabular}{cccccc}
\hline Subject & Age & Sex & $\begin{array}{c}\text { Hierarchy } \\
\text { rank }\end{array}$ & $\begin{array}{c}\text { \% of cooperation with } \\
\text { strong bond partners }\end{array}$ & $\begin{array}{c}\text { \% of cooperation with } \\
\text { weak bond partners }\end{array}$ \\
\hline Athena & Adult & Female & 14 & 82.4 & 17.6 \\
Attila & Adult & Male & 11 & 0.0 & 100.0 \\
Bart & Juvenile & Male & 22 & 76.5 & 23.5 \\
Caramello & Juvenile & Male & 22 & & \\
Clarisse & Adult & Female & 15 & 83.3 & 16.7 \\
Eliotte & Adult & Male & 9 & 53.9 & 46.1 \\
Elodie & Juvenile & Female & 22 & 67.4 & 32.6 \\
Felice & Juvenile & Male & 22 & 100.0 & 0.0 \\
Fidji & Adult & Female & 13 & 94.1 & 5.9 \\
Gaelle & Juvenile & Female & 22 & & 30.4 \\
Galack & Adult & Male & 6 & 69.6 & 19.5 \\
Hassane & Juvenile & Male & 22 & 80.5 & 6.3 \\
Luca & Juvenile & Male & 22 & 93.8 & 33.3 \\
Morticha & Juvenile & Female & 22 & 66.7 & 0.0 \\
Nelly & Adult & Female & 19 & 100.0 & 20.7 \\
Neptune & Juvenile & Female & 22 & 79.3 & 57.1 \\
Nutella & Adult & Male & 5 & 42.9 & 55.4 \\
Saana & Juvenile & Female & 22 & 44.6 & 62.8 \\
Shannon & Adult & Female & 16 & 37.2 & \\
Tamara & Juvenile & Female & 22 & 62.5 & \\
Twix & Adult & Male & 3 & 90.2 & \\
Windy & Juvenile & Female & 22 & & \\
\hline
\end{tabular}




\section{Performance}

During the test sessions, the monkeys significantly manipulated the rope when a partner was present more often after than before their first cooperation $(z=-3.91, p=0.001, N=22$; Fig. 2). Moreover, after they first cooperated, the monkeys significantly manipulated the rope in the presence of a partner more often during test sessions than during control sessions $(z=-$ 3.72, $p=0.001, N=22$; Fig. 2). During test sessions and after the monkeys first cooperated, there was no significant difference between the manipulation of the rope performed when a partner was present or absent $(z=-0.26, p=0.81, N=22$; Fig. 2$)$. However, during the test sessions and after their first cooperation, the monkeys manipulated the rope more often when a partner was in proximity (i.e. within $1.5 \mathrm{~m}$ but not in front of the rope) of the apparatus than when no partner was in proximity $(z=-3.52, p=0.001, N=22)$.

During the test sessions, the rate of success significantly increased over time (Spearman correlation: $r=0.2, p=0.02, N=150$; Fig. 3). During the control sessions, there was no significant correlation between the rate of success and session number (Spearman correlation: $r=-0.3, p=0.054, N=43 ;$ Fig. 3$).$ 


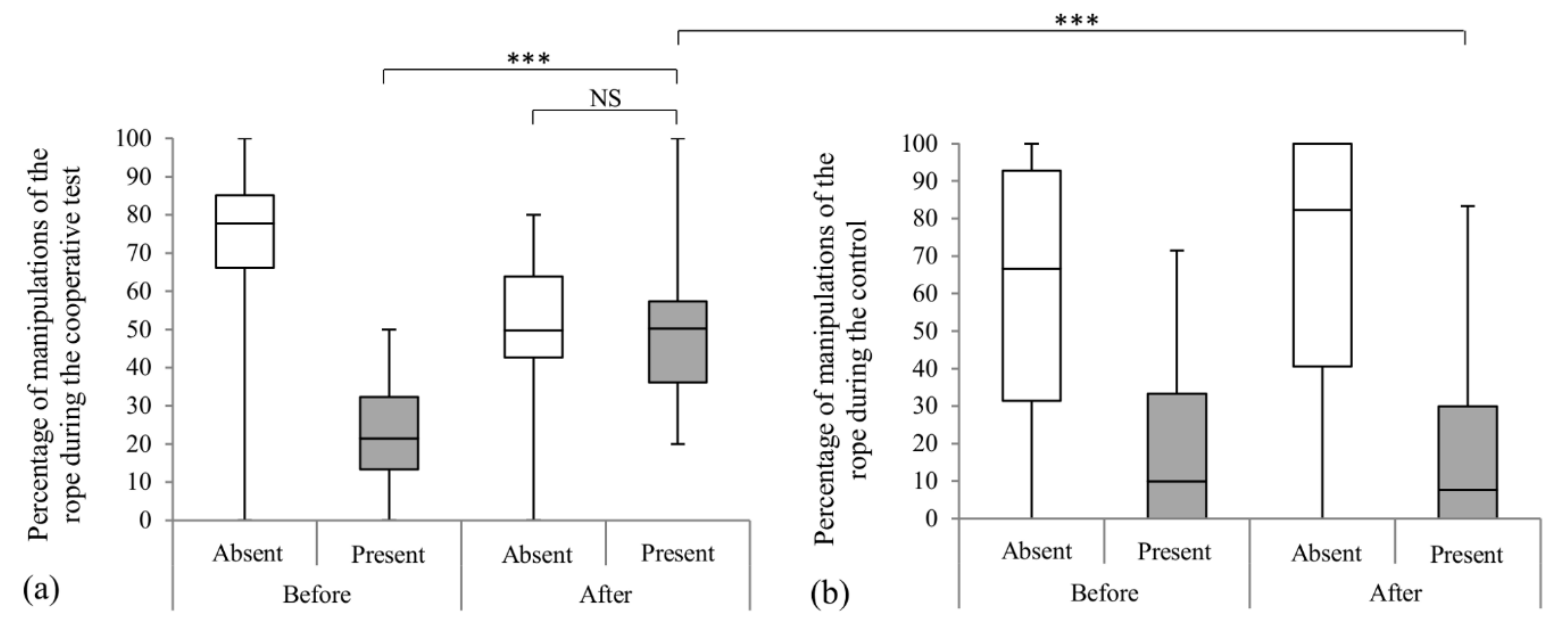

Fig. 2 For (a) the test sessions and (b) the control sessions, percentage of manipulations performed in the absence or presence of a partner, before and after the subjects' first cooperation in the test sessions $(N=22)$. The lines indicate the pairwise comparisons and their significance (NS non-significant, $* \mathrm{p}<0.05, * * \mathrm{p}<0.01, * * * \mathrm{p}<0.001)$ 

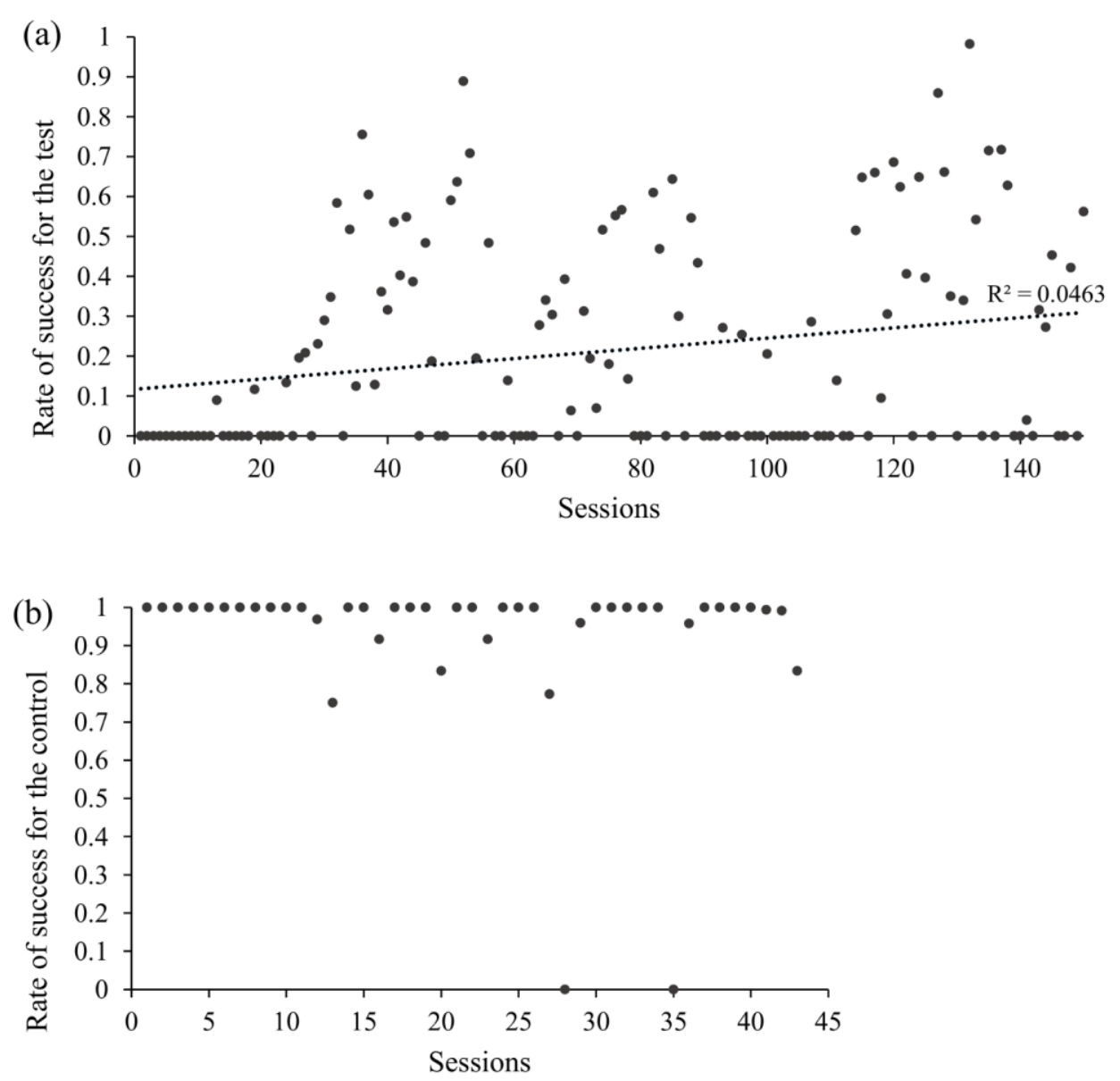

Fig. 3 Rate of success over time for (a) the test sessions $(N=150)$ and (b) for the control sessions $(N=43)$ 
Factors affecting the initiation of cooperation

The pairs who cooperated at least once had a higher tolerance level than potential pairs that never cooperated (Table 2). The pairs who cooperated at least once had also a greater difference of dominance status and were more of similar temperament, than potential pairs that never cooperated (Table 2). There was no effect of the age class, sex and strength of social bond on the likelihood of pairs to cooperate at least once (Table 2). However, because the measures of CSI and tolerance were correlated, the model was run again removing tolerance from the test variables. Following this, successful pairs had a stronger social bond than potential pairs that never cooperated (GLMM, $z=2.77, p=0.006)$, and the results of the other variables were nonsignificant (Online Resource 1).

Table 2 Coefficients and significance of the variables entered in the GLMM with a logistic distribution to test which factors affected the likelihood of pairs to cooperate at least once among the monkeys trained $(N=259)$

\begin{tabular}{ccccc}
\hline Variable & Coefficient \pm SE & $z$ & $p$ & $\mathbf{9 5 \%}$ Cis \\
\hline Strength of social bond & $-0.02 \pm 0.16$ & -0.13 & 0.9 & $-0.33-0.29$ \\
Tolerance & $70.73 \pm 19.87$ & 3.56 & 0.001 & $31.77-109.68$ \\
Rank distance & $0.14 \pm 0.07$ & 2.1 & 0.035 & $0.01-0.27$ \\
Sex of the dyad & $-0.2 \pm 0.51$ & -0.4 & 0.69 & $-1.2-0.79$ \\
Age class of the dyad & $0.68 \pm 0.78$ & 0.88 & 0.38 & $-0.84-2.21$ \\
Temperament of the dyad & $-0.01 \pm 0.01$ & -1.97 & 0.049 & $-0.03--0.0001$ \\
\hline
\end{tabular}


Factors affecting the maintenance of cooperation

Among the successful dyads, the monkeys significantly cooperated more often with partners with whom they had a strong than weak social bond $(z=-2.7, p=0.005, N=19$; Table 1; Fig. 4). Although the monkeys cooperated more with partners with whom they had a high than low level of tolerance (68.3\% of cooperation between partners of high tolerance and $31.7 \%$ between partner of low tolerance), the difference was not significant $(z=-1.73, p=0.09, N=$ 19; Fig. 4). The percentage of cooperation was not affected by the rank distance of the partners $(z=-0.65, p=0.54, N=19)$. While females cooperated more often with same-sex partners $(z=$ -2.87, $p=0.002, N=12)$, males showed no significant preference $(z=-1.36, p=0.2, N=10)$. Adults cooperated more often with juveniles than adults $(z=-2.6, p=0.006, N=10)$, whereas juveniles did not show a preference $(z=-1.87, p=0.07, N=12)$. Both shy $(z=-2.53, p=0.008$, $N=8)$ and bold monkeys $(z=-3.3, p=0.001, N=14)$ cooperated more often with bold partners.

During the control, the monkeys successfully pulled more in the absence than in the presence of a partner in front of the other box $(z=-3.13, p=0.001, N=24)$. When the monkeys pulled in the presence of another monkey in front of the other box during the control, there was no effect of social bonding $(z=-1.53, p=0.14, N=17)$, tolerance $(z=-1.52, p=0.14, N=17)$, or rank distance $(z=-0.82, p=0.44, N=17)$ on their successful pulling. Similarly, males $(z=$ $-0.42, p=0.74, N=9)$ and females $(z=-1.96, p=0.055, N=10)$ did not pulled more in the presence of same-sex or different-sex individuals, nor did age class affected the pulling of adults $(z=-0.43, p=0.77, N=10)$ and juveniles $(z=-0.14, p=0.95, N=9)$. Finally, temperament did not affect the successful pulling during the control for both bold $(z=-1.28, p=0.22, N=$ 12) and shy $(z=-1.89, p=0.13, N=7)$ monkeys. 


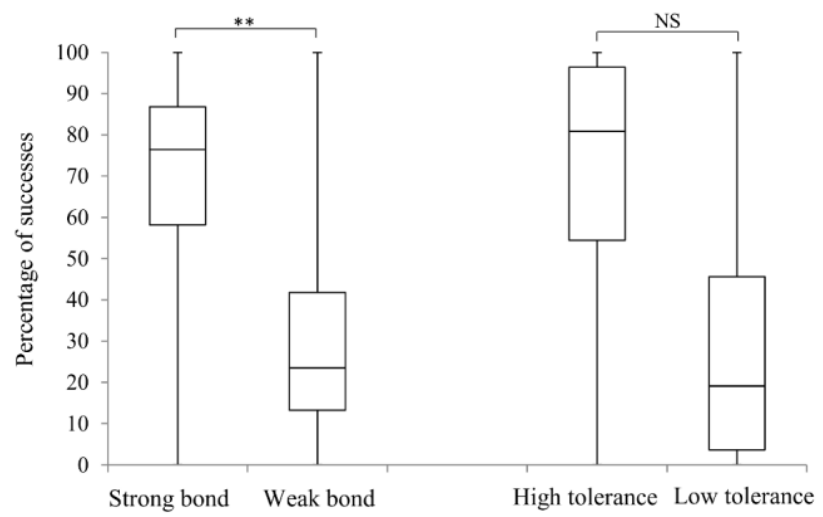

Fig. 4 Among the cooperation performed during the test sessions by each individual, percentage of cooperation performed between partners of strong or weak social bond and between partners of high or low level of tolerance $(N=19)$. The lines indicate the pairwise comparisons and their significance (NS non-significant, $* \mathrm{p}<0.05, * * \mathrm{p}<0.01, * * * \mathrm{p}<0.001$ )

\section{DISCUSSION}

This study showed that wild Barbary macaques cooperated to get food using a string pulling apparatus and that individual and social factors affected the initiation and maintenance of cooperative interactions. To our knowledge, this is the first evidence of coordinated pulling in wild macaques. Because the string was loose (Hirata and Fuwa 2007), the two ends needed to be manipulated exactly at the same time and along $75 \mathrm{~cm}$ to bring the tray within arm's reach. Consequently, the cooperation observed is unlikely to be only due to chance. Barbary macaques seem thus able to act simultaneously to reach a common goal in an experimental setting, like observed in other non-human primates (e.g. Mendres and de Waal 2000; Melis et al. 2006b; Hirata and Fuwa 2007), other mammals (Drea and Carter 2009; Plotnik et al. 2011; Möslinger et al. 2009) and birds (Seed et al. 2008; Scheid and Noë 2010; Péron et al. 2011). 
While simultaneity is an essential requirement of mutual cooperation, taking the role of the partner into account would facilitate the coordination of behaviours (Noë 2006). Our results showed that after their first cooperation, the subjects were more likely to pull when a partner was directly available, that is, when cooperation might occur. Moreover, the subjects discriminated between two different conditions that led to the same goal (i.e. obtaining food) but had different requirements in term of cooperation: the test sessions that required cooperation from a partner, and the control sessions where no cooperation was needed to reach the food. Similar results have been found, for example, in hyenas (Drea and Carter 2009) and African grey parrots (Péron et al. 2011). Discriminating between situations that required the assistance of a partner and situations that do not may allow individuals to avoid risk of conflict when a resource can be obtained alone (e.g. Melis et al. 2006a). In our study, the monkeys preferred to get the food alone when a partner was not needed during the control. The subjects may have learned the contingency between the co-occurrence of their manipulations and the food rewards through trial and error. Indeed, the rate of cooperative success increased over time during the test, indicating a learning process.

Although the subjects learned to refrain their pulling when no partner was available in front of the rope in the test sessions, they did not significantly stop this behaviour. Pulling in the absence of a partner often never fully disappears in cooperative experiments conducted on animals (e.g. Mendres and de Waal 2000; Cronin et al. 2005; Suchak et al. 2014). Several hypotheses may explain this result. First, the weak correlation coefficient between success rate and time may indicate that the learning process was slow, potentially due to the high variability of availability of partners at the apparatus. Thus after more exposures to the task, the subjects might progressively learn to precise their pulling by pulling exclusively when a partner is directly available at the rope. Second, even if the subjects continued to pull when no partner was in front of the other rope, they significantly pulled more often when a partner was in 
proximity of the apparatus than when no partner was present around the apparatus. It cannot be ruled out that pulling while being in proximity of another monkey may either be a signal to actively recruit a potential partner, by communicating their interest in obtaining the food, or may act as a local stimulus which enhanced the approach of potential partners (Jaeggi et al. 2010; Schwab et al. 2012). Indeed, communication may allow individuals to actively coordinate their actions with those of their partner by recruiting potentials partners and facilitating the coordination during the joint action. For example, visual contact has been shown to improve cooperation in capuchins (Mendres and de Waal 2000). Back glance (i.e. a side directed behaviour) is notably used by non-human primates to recruit group members for coalitions and group movement (e.g. de Waal and van Hooff 1981; Meunier et al. 2008; Sueur and Petit 2009), and may be also used during other cooperative interactions. Third, it is also possible that the monkeys were too impulsive when presented with a food reward, preventing them from totally refraining the inefficient pulling. There is evidence that many animals have difficulties to inhibit a learned response (here pulling a string) to get food (Stevens and Hauser 2004; Péron et al. 2011). Indeed, pulling requires very low energy expenditure which might explain why extraneous pulling is not inhibited completely (Suchak et al. 2014). Fourth, the fluctuation of success rate across sessions may be due to the variations of duration, trials and availability of partner across sessions. It is thus possible that the monkeys pulled alone more frequently when their preferred partners was not available, while diminishing this behaviour when their partner was available. Therefore, these results suggest that, although it is very unlikely that the cooperation observed in this experiment was achieved through random action, it remains unclear to what extent the monkeys understood the role of their partner in solving the cooperative task.

This study also showed that individual and social factors affected the cooperative interactions, but not the pulling behaviour during the control, indicating that these effects were 
specifics to the mechanisms of cooperation. Pairs of monkeys who cooperated had a higher level of tolerance than potential pairs who never cooperated, and individuals succeeded more often with partners with whom they had a strong than weak social bond. This suggests that while tolerance and relationship quality both affect cooperation, tolerance seems essential for the initiation of cooperation, whereas relationship quality may play a more important role in the maintenance of cooperation over time. These results add to the growing body of evidence showing that tolerance is necessary for the initiation of successful cooperative behaviours (e.g. Melis et al. 2006b; Hare et al. 2007). Tolerance constrained chimpanzees' cooperation even in subjects who understood the cooperative problem they had to solve (Melis et al. 2006b). Tolerance has thus an important impact on cooperative behaviour and further studies conducting cooperative task on animals should control for baseline tolerance level between subjects. In this study, dyads having a high tolerance level may have been more likely to be in proximity around the food rewards of the task, and so to have a chance to manipulate the rope at the same time, giving them the opportunity to learn the connection between their behaviour, the partner's behaviour and the food reward, facilitating further success. Moreover, there is a growing body of evidence showing that strong social bonds enhance cooperative behaviours in animals and humans (Silk 2003; Majolo et al. 2006; Schino and Aureli 2009; de Waal and Suchak 2010; Cronin 2012). Partner choice is thus key to initiate and maintain cooperative behaviours.

The temperament of the macaques, as well as their dominance status, age and sex also affected their cooperative behaviours. Females maintained cooperation more often with samesex partners. For example, in capuchins and cotton-top tamarins, same-sex partners are more efficient in solving cooperative tasks than different-sex partners (Mendres and de Waal 2000; Hauser et al. 2003). Moreover, sex of the individuals affect grooming exchange and other behaviour that requires tolerance (Mitchell and Tokunaga 1976; Lehmann and Boesch 2008). 
In particular in Barbary macaques, relationship quality is affected by the sex of the individuals; high quality social bonds being more frequent between individuals of the phylopatric sex (i.e. females; McFarland and Majolo 2011). Dyads with distant dominance rank had more chance to initiate a cooperative interaction. However, we assigned the same lowest rank to every juvenile in the group and dyads composed of two juveniles were not considered in this analysis. Thus, this result may be linked to the fact that most of the successful dyads were composed of at least one juvenile, and adults maintained cooperation more often with juveniles than with adults. Monkeys may indeed be more tolerant around food toward juveniles than adults. In Barbary macaques, adults often interact with infants and juveniles and use them as social buffer to facilitate approach and affiliation (e.g. Deag and Crook 1971; Paul et al. 1996; Kümmerli and Martin 2008). Kin relationships may also interact with dominance status during partner choice. For example, chimpanzees cooperated with partners ranking higher than themselves only if the partners were relatives (Suchak et al. 2014). Although it would have been interesting to investigate whether cooperative interactions were predicted by kin relationships between the study animals, kinship was not known for our study group. Pairs sharing a similar temperament were more compatible to initiate cooperation. Indeed, individuals of similar temperament tend to interact more often and bond together (Massen and Koski 2014). Moreover, individuals maintained cooperation more with bold than shy partners. Bolder individuals may be more likely to approach the test apparatus and attempt to solve the task, and the presence of a bolder partner may have reassured shier monkeys in attempting to solve the task (Scheid and Noë 2010). Individual temperament in term of shyness and boldness, such as the propensity to take risk or to react to stressful situation, may affect participation to cooperative interactions (e.g. Wilson et al. 1994; McNamara et al. 2004). It has been proposed that bold individuals may function as catalysers which allow shier individuals to cooperate even in situation they perceived as risky (Wilson et al. 1994; Gilby et al. 2008; Scheid and Noë 2010). Shier 
individuals would benefit from a diluted perceived risk by joining bolder individuals (Scheid and Noë 2010). This effect of temperament may thus be more important in risky cooperative interactions such as intergroup encounters where group members join forces against another group (e.g. Huntingford 1976; Heinsohn and Packer 1995; Fairbanks 2001; Nunn and Deaner 2004).

In conclusion, wild Barbary macaques were successful at pairing up with a partner to cooperate. Moreover, individual and social factors affected their choice of partner and their level of cooperation. High level of tolerance was necessary for the initiation of successful cooperation, while strong social bonds sustained cooperation over time. Sex, dominance status, age class and temperament of the subjects can also affect cooperation. So far, research on cooperation in animals has been intensively focused on the cognitive requirements for cooperation to occur. This study stresses the importance of taking individual and social factors into account when analysing cooperative interactions, in order to gain important insights into the evolutionary roots of cooperation, which is a fundamental aspect of human social behaviour. Tolerant relationships may have been a prerequisite for the evolution of cognitively complex cooperation in humans (Melis et al. 2006b; Hare 2007; Hare et al. 2007; Melis and Semmann 2010).

\section{ACKNOWLEDGMENTS}

We would like to thank the Haut Commissariat aux Eaux et Forêts et à la Lutte Contre la Désertification of Morocco for permission to conduct the research, and Professor Mohamed Qarro (Ecole Nationale Forestière d'Ingénieurs, Morocco) for his support. We are grateful to Jean-Marc Buonomano, Neal Marquez, Barbora Kuběnová and Laura Martínez Íñigo for assistance in the field. Finally, we would like to thank Sue Wiper and Trentham Monkey Forest for allowing us to pilot the use of the apparatus with the Barbary macaques housed at the park. 


\section{Compliance with Ethical Standards}

Our study complies with the ASAB Guidelines for the Treatment of Animals in Behavioural Research and Teaching. All procedures performed for this study were in accordance with the ethical standards of the University of Lincoln and of Morocco.

\section{Conflict of interest}

The authors declare that there is no conflict of interest in the conduct and reporting of this research.

\section{REFERENCES}

Altmann J (1974) Observational study of behavior: sampling methods. Behaviour 49:227-267

Balasubramaniam KN, Dittmar K, Berman CM, Butovskaya M, Cooper MA, Majolo B, Ogawa H, Schino G, Thierry B, de Waal FBM (2012) Hierarchical steepness, counter-aggression, and macaque social style scale. Am J Primatol 74:915-925

Berghänel A, Ostner J, Schröder U, Schülke O (2011) Social bonds predict future cooperation in male Barbary macaques, Macaca sylvanus. Anim Behav 81:1109-1116

Bergmüller R, Schürch R, Hamilton IM (2010) Evolutionary causes and consequences of consistent individual variation in cooperative behaviour. Phil Trans R Soc B 365:27512764

Boesch C, Boesch H (1989) Hunting behavior of wild chimpanzees in the Tai national park. Am J Phys Anthropol 78:547-573 
Burton JJ (1977) Absence de comportement coopératif spontané dans une troupe de Macaca fuscata en présence de pierres appâtées. Primates 18:359-366.

Carne C, Wiper S, Semple S (2011) Reciprocation and interchange of grooming, agonistic support, feeding tolerance, and aggression in semi-free-ranging Barbary macaques. Am $\mathbf{J}$ Primatol 73:1127-1133

Carrete M, Tella JT (2010) Individual consistency in flight initiation distances in burrowing owls: a new hypothesis on disturbance-induced habitat selection. Biol Lett 6:167-170

Carter AJ, Goldizen AW, Tromp SA (2010) Agamas exhibit behavioral syndromes: bolder males bask and feed more but may suffer higher predation. Behav Ecol 21:655-661

Chalmeau R, Gallo A (1993) Social constraints determine what is learned in the chimpanzee. Behav Proc 28:173-180

Chalmeau R, Lardeux K, Brandibas P, Gallo A (1997). Cooperative problem solving by Orangutans (Pongo pygmaeus). Int J Primatol 18:23-32

Crawford MP (1937) The cooperative solving of problems by young chimpanzees. Comparative Psychology Monographs 14:1-88

Cronin KA (2012) Prosocial behaviour in animals: the influence of social relationships, communication and rewards. Anim Behav 84:1085-1093

Cronin KA, Kurian AV, Snowdon CT (2005) Cooperative problem solving in a cooperatively breeding primate (Saguinus oedipus). Anim Behav 69:133-142

de Vries H, Netto WJ, Hanegraaf PLH (1993) Matman: a program for the analysis of sociometric matrices and behavioural transition matrices. Behaviour 125:157-175 
de Waal FBM (2000) Attitudinal reciprocity in food sharing among brown capuchin monkeys. Anim Behav 60:253-261

de Waal FBM, Davis JM (2003) Capuchin cognitive ecology: cooperation based on projected returns. Neuropsychologia 41:221-228

de Waal FBM, Suchak M (2010) Prosocial primates: selfish and unselfish motivations. Phil Trans R Soc B 365:2711-2722

de Waal FBM, van Hooff JARAM (1981) Side-directed communication and agonistic interactions in chimpanzees. Behaviour 77:164-198

Deag JM, Crook JH (1971) Social behaviour and 'agonistic buffering' in the wild Barbary macaque Macaca sylvana L. Folia Primatol 15:183-200

Drea CM, Carter AN (2009) Cooperative problem solving in a social carnivore. Anim Behav 78:967-977

Fady JC (1972) Absence de coopération de type instrumental en milieu nature1 chez Papio papio. Behaviour 93:157-164.

Fairbanks LA (2001) Individual differences in response to a stranger: social impulsivity as a dimension of temperament in vervet monkeys (Cercopithecus aethiops sabaeus). J Comp Psychol 115:22-28

Gilby IC, Eberly LE, Wrangham RW (2008) Economic profitability of social predation among wild chimpanzees: individual variation promotes cooperation. Anim Behav 75:351-360

Hare B (2007) From nonhuman to human mind: what changed and why. Curr Dir Psychol Sci $16: 60-64$ 
Hare B, Melis AP, Woods V, Hastings S, Wrangham RW (2007) Tolerance allows bonobos to outperform chimpanzees on a cooperative task. Curr Biol 17:619-623

Hattori Y, Kuroshima H, Fujita K (2005) Cooperative problem solving by tufted capuchin monkeys (Cebus apella): spontaneous division of labor, communication, and reciprocal altruism. J Comp Psychol 119:335-342

Hauser MD, Chen MK, Chen F, Chuang E (2003) Give unto others: genetically unrelated cotton-top tamarin monkeys preferentially give food to those who altruistically give food back. Proc R Soc B 270:2363-2370

Heinsohn R, Packer C (1995) Complex cooperative strategies in group-territorial African lions. Science 269:1260-1262

Hirata S, Fuwa K (2007) Chimpanzees (Pan troglodytes) learn to act with other individuals in a cooperative task. Primates 48:13-21

Huntingford FA (1976) The relationship between anti-predator behaviour and aggression among conspecifics in the three-spined stickleback, Gasterosteus aculeatus. Anim Behav $24: 245-260$

Jaeggi AV, Burkart JM, van Schaik CP (2010) On the psychology of cooperation in humans and other primates: combining the natural history and experimental evidence of prosociality. Phil Trans R Soc B 365:2723-2735

Kaburu SSK, MacLarnon A, Majolo B, Qarro M, Semple S (2012) Dominance rank and selfscratching among wild female Barbary macaques (Macaca sylvanus). Afr Zool 47:74-79

Kümmerli R, Martin RD (2008) Patterns of infant handling and relatedness in Barbary macaques (Macaca sylvanus) on Gibraltar. Primates 49:271-282 
Lehmann J, Boesch C (2008) Sex differences in chimpanzee sociality. Int J Primatol 29:65-81

Majolo B, Ames K, Brumpton R, Garratt R, Hall K, Wilson N (2006) Human friendship favours cooperation in the iterated Prisoner's Dilemma. Behaviour 143:1383-1395

Maréchal L, Semple S, Majolo B, Qarro M, MacLarnon A (2011) Impacts of tourism on anxiety and physiological stress in wild male Barbary macaques. Biol Cons 144:2188-2193

Massen JJM, Koski1, SE (2014) Chimps of a feather sit together: chimpanzee friendships are based on homophily in personality. Evol Hum Behav 35:1-8

McFarland R, Majolo B (2011). Exploring the components, asymmetry and distribution of relationship quality in wild Barbary macaques (Macaca sylvanus). PLoS ONE 6:e28826

McNamara JM, Barta Z, Houston AI (2004) Variation promotes cooperation in the Prisoner's Dilemma game. Nature 428:745-747

McNamara JM, Leimar O (2010) Variation and the response to variation as a basis for successful cooperation. Phil Trans R Soc B 365:2627-2633

Melis AP, Hare B, Tomasello M (2006a) Chimpanzees recruit the best collaborators. Science $311: 1297-1300$

Melis AP, Hare B, Tomasello M (2006b) Engineering cooperation in chimpanzees: tolerance constraints on cooperation. Anim Behav 72:275-286

Melis AP, Semmann D (2010) How is human cooperation different? Phil Trans R Soc B $365: 2663-2674$

Melis AP, Tomasello M (2013) Chimpanzees' (Pan troglodytes) strategic helping in a collaborative task. Biol Lett 9:20130009 
Mendres KA, de Waal FBM (2000) Capuchins do cooperate: the advantage of an intuitive task. Anim Behav 60:523-529

Meunier H, Deneubourg JL, Petit O (2008) How many for dinner? Recruitment and monitoring by glances in capuchins. Primates 49:26-31

Mitchell G, Tokunaga DH (1976) Sex differences in nonhuman primate grooming. Behav Proc $4: 335-345$

Møller AP, Nielsen JT, Garamzegi LZ (2008) Risk taking by singing males. Behav Ecol 19:4153

Möslinger H, Kotrschal K, Huber L, Range F, Virányi Z (2009) Cooperative string-pulling in wolves. J Vet Behav 4:99

Noë R (2001) Biological markets: partner choice as the driving force behind the evolution of cooperation. In: Noë R, van Hooff JARAM, Hammerstein P (ed) Economics in nature: social dilemmas, mate choice and biological markets. Cambridge University Press, Cambridge, pp. 93-118

Noë R (2006) Cooperation experiments: coordination through communication versus acting apart together. Anim Behav 71:1-18

Noldus Information Technology (2003) Matman, software for matrix manipulation and analysis, version 1.1. Wageningen, The Netherlands

Nunn CL, Deaner RO (2004) Patterns of participation and free riding in territorial conflicts among ringtailed lemurs (Lemur catta). Behav Ecol Sociobiol 57:50-61

Pansini R (2011) Induced cooperation to access a sharable reward increases the hierarchical segregation of wild vervet monkeys. PLoS ONE 6:e21993 
Paul A, Kuester J, Arnemann J (1996). The sociobiology of male-infant interactions in Barbary macaques (Macaca sylvanus). Anim Behav 51:155-170

Pereira ME, Fairbanks LA (2002) Juvenile primates: life history, development and behavior, with a new foreword. University of Chicago Press, Chicago

Péron F, Rat-Fischer L, Lalot M, Nagle L, Bovet D (2011) Cooperative problem solving in African grey parrots (Psittacus erithacus). Anim Cogn 14:545-553

Petit O, Desportes C, Thierry B (1992) Differential probability of 'coproduction' in two species of macaque (Macaca tonkeana, M. mulatta). Ethology 90:107-120

Pinheiro JC, Bates DM (2000) Mixed effects models in S and S-PLUS, 1st edn. SpringerVerlag, New York

Plotnik JM, Lair R, Suphachoksahakun W, de Waal FBM (2011) Elephants know when they need a helping trunk in a cooperative task. Proc Natl Acad Sci USA 108:5116-5121

Scheid C, Noë R (2010) The performance of rooks in a cooperative task depends on their temperament. Anim Cogn 13:545-553

Schino G, Aureli F (2009) Reciprocal altruism in primates: partner choice, cognition and emotions. Adv Stud Behav 39:45-69

Schwab C, Swoboda R, Kotrschal K, Bugnyar T (2012) Recipients affect prosocial and altruistic choices in jackdaws, Corvus monedula. PLoS ONE, 7, e34922

Seed AM, Clayton NS, Emery NJ (2008) Cooperative problem solving in rooks (Corvus frugilegus). Proc R Soc B 275:1421-1429

Silk JB (2003) Cooperation without counting: the puzzle of friendship. In: Hammerstein P (ed) The genetic and cultural evolution of cooperation. The MIT Press, Cambridge, pp 37-54 
Silk JB (2006) Practicing Hamilton's rule: kin selection in primate groups. In: Kappeler PM, van Schaik CP (ed) Cooperation in primates and humans: mechanisms and evolution. Springer, New-York, pp 25-46

Silk JB, Altmann J, Alberts S (2006) Social relationships among adult female baboons (Papio cynocephalus) I. Variation in the strength of social bonds. Behav Ecol Sociobiol 61:183195

Stankowich T, Coss RG (2005) Effects of predator behavior and proximity on risk assessment by Columbian black-tailed deer. Behav Ecol 17:246-254

Stevens JR, Hauser MD (2004) Why be nice? Psychological constraints on the evolution of cooperation. Trends Cogn Sci 8:60-65

Suchak M, Eppley TM, Campbell MW, de Waal FBM (2014) Ape duos and trios: spontaneous cooperation with free partner choice in chimpanzees. PeerJ 2:e417

Sueur C, Petit O (2009) Signals use by leaders in Macaca tonkeana and Macaca mulatta: groupmate recruitment and behaviour monitoring. Anim Cogn 13:239-248

Thierry B, Singh M, Kaumanns W (2004) Macaque societies: a model for the study of social organization. Cambridge University Press, Cambridge

Widdig A, Streich W, Tembrock G (2000) Coalition formation among male Barbary macaques (Macaca sylvanus). Am J Primatol 50:37-51

Wilson DS, Clark AB, Coleman K, Dearstyne T (1994) Shyness and boldness in humans and other animals. Trends Ecol Evol 9:442-446 
Supplementary material: Online Resource 1

Table 1 Coefficients and significance of the variables entered in the GLMM with a logistic distribution to test which factors affected the likelihood of pairs to cooperate at least once among the monkeys trained, when removing the variable 'tolerance' $(N=259)$

\begin{tabular}{ccccc}
\hline Variable & Coefficient \pm SE & $z$ & $p$ & 95\% CIs \\
\hline Strength of social bond & $0.34 \pm 0.12$ & 2.77 & 0.006 & $0.1-0.59$ \\
Rank distance & $0.12 \pm 0.06$ & 1.81 & 0.07 & $-0.01-0.24$ \\
Sex of the dyad & $-0.39 \pm 0.48$ & -0.8 & 0.42 & $-1.33-0.56$ \\
Age class of the dyad & $0.76 \pm 0.82$ & 0.92 & 0.36 & $-0.85-2.36$ \\
Temperament of the dyad & $-0.01 \pm 0.01$ & -1.78 & 0.075 & $-0.03-0.001$ \\
\hline
\end{tabular}

